\title{
A Cellular Construction of BP and Other Irreducible Spectra
}

\author{
Stewart Priddy \\ Department of Mathematics, Northwestern University, Evanston, Illinois 60201, U.S.A.
}

In this note we construct and derive the basic properties of the Brown-Peterson spectrum BP by attaching cells to the sphere spectrum $S^{0}$ (localized at a prime p) so as to kill the odd-dimensional homotopy groups. This procedure is, of course, entirely analogous to the construction of the Eilenberg-MacLane spectrum $K\left(\mathbb{Z}_{(p)}\right)$ by killing the positive dimensional homotopy groups of $S^{0}$. One can thus view BP as lying "half-way" between $S^{0}$ and $K\left(\mathbb{Z}_{(p)}\right)$. The advantage of our approach is that it avoids computations with Steenrod operations inherent in the original Postnikov tower construction [2]. Moreover, we obtain the homotopy and cohomology groups of BP immediately from the construction by a simple application of obstruction theory and the Adams spectral sequence.

Pedagogically, we have found this approach useful in introducing BP to students who have mastered homotopy theory including the Adams spectral sequence, as for example from the texts of Moser-Tangora [5] or Switzer [9].

This paper consists of four sections the first of which gives the construction of our candidate $X$ for the BP spectrum after making precise the notion of attaching cells "non-trivially". $X \simeq \mathrm{BP}$ follows easily from the fact that a self map of a complex with cells attached non-trivially is an equivalence iff it is an equivalence on the bottom cell. In $\S 2$ we derive the main properties of $X$ directly from the construction without assuming the existence of BP. The idea is to prove $H^{*}(X ; \mathbb{Z} / p)$ is free over $A /(\beta)$, then use the Adams spectral sequence to simultaneously compute $\pi_{*} X$ and show $H^{*}(X ; \mathbb{Z} / p)$ is monogenic. In $\S 3$ we analyze the individual spaces of our spectrum. They are found to be equivalent to certain spaces in Wilson's spectrum $\mathrm{BP}\langle n\rangle$. Finally in $\S 4$ we show that by attaching cells to kill the $(4 k-1)$ dimensional homotopy groups of $S^{0}$ we obtain an interesting spectrum which is equivalent to $M S p$ through the 30 -skeleton.

Recollections. For convenience we recall the following properties of $\mathrm{BP}$ from [2]:

i) $\pi_{*} \mathrm{BP}=\mathbb{Z}_{(p)}\left[v_{1}, v_{2}, \ldots\right],\left|v_{i}\right|=2\left(p^{i}-1\right)$

ii) $H^{*}(\mathrm{BP} ; \mathbb{Z} / p)=A /(\beta)$, where $A$ is the $\bmod -p$ Steenrod algebra and $(\beta)$ is the two-sided ideal generated by the Bockstein. 
Conventions. Throughout this paper, we work in the category of $p$-local CWcomplexes (spectra) with basepoint. All modules are finitely generated over $\mathbb{Z}_{(p)}$, the integers localized at the prime $p$.

\section{$\S 1$. Construction}

Definition. Given spaces $A \subset Z$, we say that $Z$ is obtained from $A$ by non-trivially attaching $(n+1)$-cells if $Z=A \cup \bigcup_{f_{\alpha}} e_{\alpha}^{n+1}$ for some set of attaching maps
$\left\{f_{\alpha}: S^{n} \rightarrow A\right\}$ such that

where

$$
\operatorname{ker}\left(\bigoplus_{\alpha} f_{\alpha^{*}}\right) \subset p \cdot\left(\bigoplus_{\alpha} \pi_{n} S^{n}\right)
$$

$$
\bigoplus_{\alpha} f_{\alpha^{*}}: \bigoplus_{\alpha} \pi_{n} S^{n} \rightarrow \pi_{n} A
$$

is given by summation. Equivalently, if $\operatorname{dim} A \leqq n$, one can require that every element of $\pi_{n+1} Z$ have trivial Hurewicz image $\bmod p$ (this point is elucidated in the proof of the theorem in $\$ 2$ ).

Similarly one can attach stable cells non-trivially in the stable category of $p$ local CW-spectra.

Construction. We can now define our candidate $X$ for the BP spectrum. For $n \geqq 0$, let $X_{n}$ be obtained from $S^{n}$ by non-trivially attaching cells in dimensions $n$ $+2, n+4, \ldots, n+2 k, \ldots$ to kill the homotopy groups in dimensions $n+1, n$ $+3, \ldots, n+2 k-1, \ldots$ That is, if $\left(X_{n}\right)^{k}$ denotes the $k$-skeleton then $\left(X_{n}\right)^{n}=\left(X_{n}\right)^{n+1}$ $=S^{n}$ and assuming $\left(X_{n}\right)^{n+2 k-1}$ to be defined we attach $(n+2 k)$-cells non-trivially to obtain $\left(X_{n}\right)^{n+2 k}$ so that $\pi_{n+2 k-1}\left(\left(X_{n}\right)^{n+2 k}\right)=0$.

Structure maps $\varepsilon_{n}: X_{n} \rightarrow \Omega X_{n+1}$ are easy to define. By construction $\pi_{n+2 k-1} \Omega X_{n+1}=\pi_{n+2 k} X_{n+1}=0$, hence $H^{n+2 k}\left(X_{n} ; \pi_{n+2 k-1} \Omega X_{n+1}\right)=0$ and so by obstruction theory there exists a map $\varepsilon_{n}: X_{n} \rightarrow \Omega X_{n+1}$ extending the identity map on $S^{n}$. Thus $X=\left\{X_{n}, \varepsilon_{n}\right\}$ is a spectrum.

Presently in $\S 2$, we shall show $X$ has the desired properties without assuming BP exists. For now, we show we have made the proper construction.

Theorem. $X$ is homotopy equivalent to BP.

Definition. A space (spectrum) $Z$ is called irreducible if it is obtained from a sphere (sphere spectrum) $S^{N}$ by successively attaching cells (stable cells) nontrivially.

Proposition. Suppose $Z$ is irreducible (and simply connected if $Z$ is a space). Then $\mathrm{g}: Z \rightarrow Z$ is a homotopy equivalence if $\left.\mathrm{g}\right|_{S^{N}}$ is a homotopy equivalence.

Proof. We inductively assume $g: Z^{n} \rightarrow Z^{n}$ is an equivalence. Consider the commutative diagram derived from the cofibration $V S^{n} \rightarrow Z^{n} \rightarrow Z^{n+1}$ $\left(H_{*}=H_{*}\left(\cdot ; \mathbb{Z}_{(p)}\right)\right)$ 


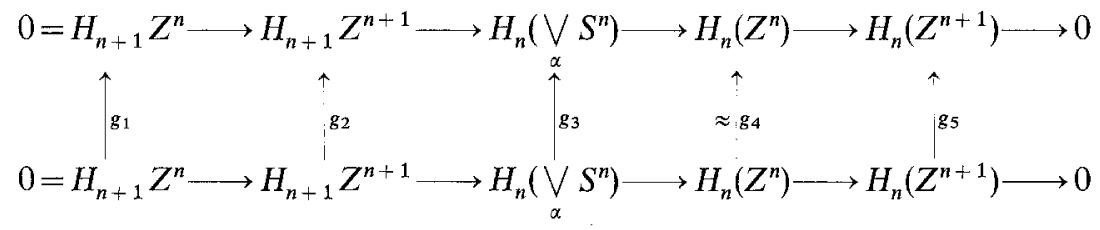

where $g_{i}$ is induced by $g$. Since $Z$ is simply connected, it suffices to show $g_{2}$ is an automorphism. By assumption $g_{4}$ and hence $g_{5}$ is an automorphism (for $g_{5}$ we use the well known fact that for a finitely generated module any epic endomorphism is bijective). Thus $g_{2}$ is bijective iff $g_{3}$ is. Using the Hurewicz homomorphism we are reduced to showing $g_{3}$ is an automorphism in homotopy. Consider the commutative diagram where $g_{4}$ is bijective by assumption.

Thus we have
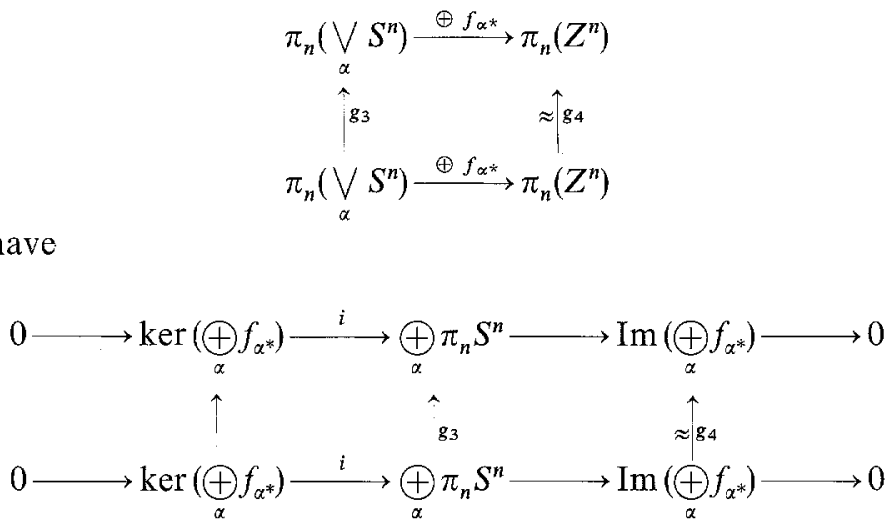

Tensoring with $\mathbb{Z} / p$ and using the definition of irreducible we have $i \otimes \mathbb{Z} / p=0$. Thus $g_{3} \otimes \mathbb{Z} / p$ is bijective. By Nakayama's lemma $g_{3}$ is bijective, which completes the proof.

Proof of the Theorem. Since $\pi_{2 k-1} X=\pi_{2 k-1} \mathrm{BP}=0=H^{2 k-1} \mathrm{BP}=H^{2 k-1} X$, obstruction theory gives maps $g: X \rightarrow \mathrm{BP}, h: \mathrm{BP} \rightarrow X$ extending the identity on $S^{0}$. By the proposition, $h g: X \rightarrow X$ is therefore an equivalence. Since $H^{*}(\mathrm{BP} ; \mathbb{Z} / p)$ $=A /(\beta)$ is monogenic, $g h: \mathrm{BP} \rightarrow \mathrm{BP}$ is also an equivalence.

\section{§ 2. Properties}

In this section we derive the main properties of the spectrum $X$ directly from the construction of $\S 1$.

Theorem. i) $\pi_{*} X=\mathbb{Z}_{(p)}\left[v_{1}, v_{2}, \ldots\right],\left|v_{i}\right|=2\left(p^{i}-1\right)$,

ii) $H^{*}(X ; \mathbb{Z} / p)=A /(\beta)$.

First we observe that $X$ is a ring spectrum with unit since by obstruction theory there exists maps $X_{m} \wedge X_{n} \rightarrow X_{m+n}$ extending the usual homeomorphism $S^{m} \wedge S^{n} \rightarrow S^{m+n}$. 
Lemma. $H^{*}(X ; \mathbb{Z} / p)$ is free over $A /(\beta)$.

We give two proofs of this fact. The first uses the cohomology of the complex cobordism spectrum $M U$; the second is direct from the construction using the Nishida formula.

First Proof. Since the cohomology of $M U$ is even dimensional, obstruction theory gives a map $f: M U \rightarrow X$ extending the identity on $S^{0}$. Let $l: X \rightarrow K\left(\mathbb{Z}_{(p)}\right)$ classify $1 \in H^{0} X$. Since $H^{*}(M U ; \mathbb{Z} / p)$ is free over $A /(\beta)$ (see [1; II 8.4]) the composite $M U \stackrel{f}{\longrightarrow} X \stackrel{\imath}{\longrightarrow} K\left(\mathbb{Z}_{(p)}\right)$ induces a monomorphism in mod-p cohomology. Since $l$ is a map of ring spectra the Milnor-Moore theorem shows $H^{*}(X ; \mathbb{Z} / p)$ is free over $A /(\beta)$.

Second Proof. We shall show that evaluation on the unit $A /(\beta) \rightarrow H^{*}(X ; \mathbb{Z} / p)$ is injective. Let $\alpha_{1} \in \pi_{2 p-3} S^{0}$ be a generator and consider $Y=S^{0} \bigcup_{\alpha_{1}} e^{2 p-2} \rightarrow X$ extending the unit map $S^{0} \rightarrow X\left(\alpha_{1}=\eta\right.$ if $\left.p=2\right)$. By obstruction theory there are maps $D_{p}\left(X_{2 n}\right)=\left(E \Sigma_{p^{+}}\right) \bigwedge_{\Sigma_{p}}\left(X_{2 n}\right)^{(p)} \rightarrow X_{2 p n}$ extending the ring structure maps $\left(X_{2 n}\right)^{(p)} \rightarrow X_{2 p n}$ (the cohomology of $D_{p}\left(X_{2 n}\right)$ is even dimensional). Hence we may form the composite $D_{p}\left(\ldots\left(D_{p} Y\right) \ldots\right) \rightarrow D_{p}\left(\ldots\left(D_{p} X\right) \ldots\right) \rightarrow X$ and by naturality we are reduced to checking that Milnor's element $P^{\Delta_{i}}\left(S q^{2 \Delta_{i}}\right.$ if $\left.p=2\right)$ is non-zero on the bottom class of $D_{p}\left(\ldots\left(D_{p} Y\right) \ldots\right)$. But this verification follows using the Nishida formula as in Proposition 3.4 of [6].

Proof of the Theorem. We shall prove i) and ii) simultaneously using the Adams spectral sequence

$$
E_{2}^{s, t}=\operatorname{Ext}_{A}^{s, t}\left(H^{*}(X ; \mathbb{Z} / p), \mathbb{Z} / p\right) \Rightarrow \pi_{t-s} X .
$$

By the lemma, $H^{*}(X ; \mathbb{Z} / p)$ is free over $A /(\beta)$ on even dimensional generators say $\left\{g_{\gamma}\right\}$. Thus $H^{*}(X ; \mathbb{Z} / p)=\bigoplus_{\gamma}[A /(\beta)] g_{\gamma}$. According to Milnor $[4], A /(\beta)=A \underset{E}{\otimes} \mathbb{Z} / p$ where $E=E\left[Q_{0}, Q_{1}, \ldots\right],{ }^{\gamma} Q_{0}=\beta, Q_{i+1}=\left[\mathscr{P}^{p^{i}}, Q_{i}\right],\left|Q_{i}\right|=2 p^{i}-1$. Thus by change of rings and the standard formula for the cohomology of an exterior algebra we have

$$
\begin{aligned}
E_{2} & =\underset{\gamma}{\oplus} \operatorname{Ext}_{A}^{s_{i} t}(A /(\beta), \mathbb{Z} / p) \cdot g_{\gamma}=\underset{\gamma}{\oplus} \operatorname{Ext}_{E}^{s, t}(\mathbb{Z} / p, \mathbb{Z} / p) \cdot g_{\gamma} \\
& =\oplus_{\gamma} \mathbb{Z} / p\left[v_{0}, v_{1}, v_{2}, \ldots\right] \cdot g_{\gamma} \quad \text { with }\left|v_{i}\right|=2\left(p^{i}-1\right) .
\end{aligned}
$$

Also $E_{2}=E_{\infty}$ by even dimensionality. Now we claim there is only one generator $g_{\gamma}$ and it is in dimension zero. Suppose $g_{\gamma}$ is a generator of minimal positive dimension $2 n$. Then $g_{\gamma} \in \operatorname{Ext}^{0,2 n}\left(H^{*}(X ; \mathbb{Z} / p), \mathbb{Z} / p\right)$ represents a class in $\pi_{2 n} X$ which has non-zero Hurewicz image $\bmod p$. Since $\pi_{2 n} X \approx \pi_{2 n} X^{2 n}$ we have $h j\left(g_{\gamma}\right) \neq 0$ in

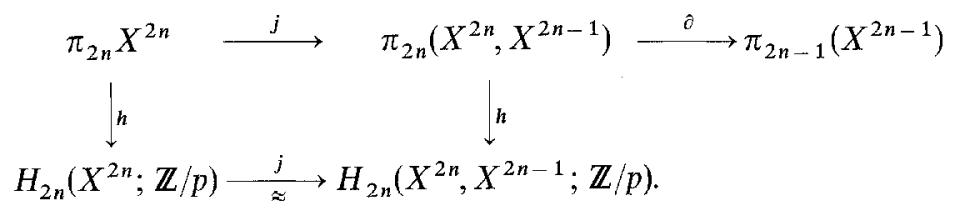


However $\partial j\left(g_{y}\right)=0$ by exactness so $\operatorname{ker} \partial \notin p \cdot \pi_{2 n}\left(X^{2 n}, X^{2 n-1}\right)$ contradicting the irreducibility of $X$. Thus $H^{*}(X ; \mathbb{Z} / p)=A /(\beta)$ and $\pi_{*} X=\mathbb{Z}_{(p)}\left[v_{1}, v_{2}, \ldots\right]$ since $v_{0}$ corresponds to multiplication by $p$. Q.E.D.

\section{$\S 3$. The Spaces $X_{n}$}

In this section we identify the individual spaces $X_{n}$ of the spectrum $X$ of $\S 1$.

Using the techniques of Baas-Sullivan, Wilson [10] has constructed $\Omega$ spectra $\mathrm{BP}\langle n\rangle=\left\{\mathrm{BP}\langle n\rangle_{k}\right\}$ for $0 \leqq n \leqq \infty$ such that $\mathrm{BP}\langle 0\rangle=K\left(\mathbb{Z}_{(p)}\right), \mathrm{BP}\langle\infty\rangle$ $=\mathrm{BP}$ and $\pi_{*} \mathrm{BP}\langle n\rangle=\mathbb{Z}_{(p)}\left[v_{1}, v_{2}, \ldots, v_{n}\right]$.

Lemma. (Wilson $[10 ; 5.1,6.9])$. i) for $k \leqq\left(p^{n}+\ldots+p+1\right), H^{*}\left(\mathrm{BP}\langle n\rangle_{2 k} ; Z_{(p)}\right)$ is $a$ polynomial algebra on even dimensional classes.

ii) for $k>\left(p^{n-1}+\ldots+p+1\right)$, any map $\mathrm{BP}\langle n\rangle_{k} \rightarrow \mathrm{BP}\langle n\rangle_{k}$ which induces an isomorphism on $\pi_{k}$ is a homotopy equivalence.

Proposition. If $2\left(p^{n-1}+\ldots+p+1\right)<2 k \leqq 2\left(p^{n}+\ldots+p+1\right)$ then $X_{2 k}$ is homotopy equivalent to $\mathrm{BP}\langle n\rangle_{2 k}$.

Proof. By part i) of the lemma and obstruction theory there are maps $f: \mathrm{BP}\langle n\rangle_{2 k} \rightarrow X_{2 k}$ and $g: X_{2 k} \rightarrow \mathrm{BP}\langle n\rangle_{2 k}$ extending the identity on $S^{2 k}$. Since $X_{2 k}$ is irreducible $f g$ is an equivalence. Similarly $g f$ is an equivalence by part ii) of the lemma.

Example. For $p=2$, we have $X_{2} \simeq \mathrm{BP}\langle 0\rangle_{2} \simeq \mathbb{C} P^{\infty}, \quad X_{4} \simeq \mathrm{BP}\langle 1\rangle_{4} \simeq B S U$, $X_{6} \simeq \mathrm{BP}\langle 1\rangle_{6} \simeq B S U[6, \ldots]$. The element $v_{n}$ first appears on the $2^{n+1}$ sphere, i.e. $X_{2^{n+1}} \simeq \mathrm{BP}\langle n\rangle_{2^{n+1}}$ while $X_{2^{n+1}-2} \simeq \mathrm{BP}\langle n-1\rangle_{2^{n+1}-2}$.

\section{§4. Other Irreducible Ring Spectra}

In this section we consider the spectrum $Y$ obtained from the sphere spectrum $S^{0}$ by killing $\pi_{4 k-1}, k \geqq 1$. This spectrum is related to the symplectic cobordism spectrum $M S p$; however, as in the case of $M S p$, our information about $Y$ is incomplete. Of course, $X$ and $Y$ are part of a family of irreducible ring spectra (one for each $N \geqq 1$ ) obtained from $S^{0}$ by killing $\pi_{2^{N-1}}, k \geqq 1$. These spectra approximate $S^{0}$ as $N \rightarrow \infty$ and so one expects their properties to become progressively more intractable.

Construction. The spaces $Y_{n}$ of the spectrum $Y$ are obtained from $S^{n}$ by successively attaching cells non-trivially in dimensions $n+4, n+8, \ldots, n+4 k, \ldots$ so as to kill the homotopy in dimensions $n+3, n+7, \ldots, n+4 k-1, \ldots$. As in the case of $X$, obstruction theory allows us to define maps $Y_{n} \rightarrow \Omega Y_{n+1}$, $Y_{m} \wedge Y_{n} \rightarrow Y_{m+n}$ and so $Y$ is a ring spectrum.

Remark. Since $\pi_{7} S^{4}=\mathbb{Z} \oplus Z / 12$, one can show $Y_{4} \simeq B S p$ for $p=2,3$.

Since $H^{*}(M S p ; \mathbb{Z} / p)$ is zero in dimensions not divisible by 4 , there is a map $f: M S p \rightarrow Y$ extending the identity on $S^{0}$. 
Lemma. $H^{*}(Y ; \mathbb{Z} / p)$ is free over $A /(\beta)\left(\right.$ over $A^{\prime}=\mathbb{Z} / 2\left[\xi_{1}^{4}, \xi_{2}^{4}, \ldots\right]^{*}$ if $\left.p=2\right)$.

Proof. Let $\imath: Y \rightarrow K\left(\mathbb{Z}_{(p)}\right)$ classify $1 \in H^{0} Y$. Then arguing as in the first proof of the lemma of $\S 2$ and using the fact that $H^{*}(M S p ; \mathbb{Z} / p)$ is free over $A /(\beta)$ (over $A^{\prime}$ if $p$ $=2$ ) we have the desired result.

Corollary. $Y$ is homotopy equivalent to BP for $p>2$.

Henceforth we assume $p=2$. Now using results of Kochman [3], Ray [7], and Segal [8] we can compare $Y$ and $M S p$ more closely.

Proposition. $f: M S p \rightarrow Y$ is a homotopy equivalence through dimension 30 .

Proof. $M S p$ is irreducible through the 28 skeleton and $\pi_{4 k-1} M S p=0$ for $k<8$ $[8,3]$. Hence there is a map $g: Y^{30} \rightarrow M S p^{30}$ such that $g f$ and $f g$ are equivalences on the 30-skeleton. Q.E.D.

It is interesting to note the related facts: $\pi_{31} M S p \neq 0[7,3]$ and $M_{S p}{ }^{32}$ is not irreducible [3]. If $x_{2} \in H^{8}(M S p ; \mathbb{Z} / 2)$ denotes an indecomposable element over $A^{\prime}$ then $x_{2}^{4}$ represents a non-zero element of $\pi_{32} M S p$ with non-zero Hurewicz image $\bmod 2$. In fact this is the first positive dimensional element with non-zero image in $\pi_{*} M S p \rightarrow \pi_{*} M O$; its image is $\left(\mathbb{R} P^{2}\right)^{16}[8]$.

\section{References}

1. Adams, J.F.: Stable homotopy and generalized cohomology. Chicago: University of Chicago Press 1974

2. Brown, E., Peterson, F.: A spectrum whose $\mathbb{Z}_{p}$ cohomology is the algebra of reduced $p^{\text {th }}$ powers. Topology 5, 149-154 (1966)

3. Kochman, S.: The symplectic cobordism ring I. Mem. Amer. Math. Soc. 228 (1980)

4. Milnor, J.: The Steenrod algebra and its dual. Ann. of Math. 67, 150-171 (1958)

5. Mosher, R., Tangora, M.: Cohomology operations and applications in homotopy theory. New York: Harper and Row 1968

6. Nishida, G.: The nilpotency of elements of the stable homotopy groups of spheres. J. Math. Soc. Japan 25, 707-732 (1973)

7. Ray, N.: Indecomposables in Tors $M S p_{*}$. Topology 10, 261-270 (1971)

8. Segal, D.: On the symplectic cobordism ring. Comment Math. Helv. 45, 159-169 (1970)

9. Switzer, R.: Algebraic topology-homotopy and homology. Berlin-Heidelberg-New York: Springer 1975

10. Wilson, S.: The $\Omega$-spectrum for Brown-Peterson cohomology part II. Amer. J. Math. 97, 101-123 (1975) 